\title{
A descriptive study to assess the drug sensitivity pattern to antitubercular drugs in patients visiting department of respiratory medicine at a tertiary care hospital at Western Maharashtra
}

\author{
HS Sndhu', Shishir Jain 2,*, Ashish Bahal', Ayyappa G ${ }^{4}$ \\ ${ }^{1,2,3}$ Assistant Professor, ${ }^{4}$ Resident, ${ }^{1,3}$ Dept. of Pathology, Military Hospital, (CTC), Pune, ${ }^{2,4}$ Dept. of Community Medicine, \\ Armed Forces Medical College, Pune, Maharashtra, India
}

*Corresponding Author:

Email: shishirjainpro@gmail.com

\begin{abstract}
Objective/Introduction: Tuberculosis is a overwhelming public health worry in India. The Indian Subcontinent accounts for about $25 \%$ of the TB burden worldwide. Across the world, India has the highest burden of both TB and MDR-TB. The aim of this study was to study the resistance pattern of_Mycobacterium Tuberculosis to first and second line anti-TB drugs_among specimens from a hospital in Tertiary Care Hospital in Western Maharashtra.

Materials and Method: This record based study was conducted at Tertiary Care Hospital in Western Maharashtra,

from January 2017 .Records of Sputum from 2013 to 2017 were obtained from patients with suspected TB. Drug-sensitivity testing was performed by the Mycobacteria Growth Indicator Tube (MGIT) method. Sensitivity to First line and second line drugs and Quinolones were tested.

Results: Among 734 patients, 701(95.5\%) were males and 33(4.5\%) were females. Analysis of our Study result showed that $21.7 \%$ of patients were mono-drug resistant. Out of 734 isolates, $56(7.62 \%)$ showed multi-drug resistance (resistance to INH and RIF at least) and 33 out of $734(4.5 \%)$ whereas 39 (5.6\%) out of the 734 samples showed XDR.

Conclusion: This study confirms that drug resistance, including MDR, observed against all first-line TB drugs was a real threat in the management of TB infection. The patterns of resistance in this study will assist clinicians in providing the right treatment regimen to TB patients and bring about an improvement in their clinical condition.
\end{abstract}

Keywords: Pulmonary Tuberculosis, Drug resistance.

\section{Introduction}

Tuberculosis (TB) is an infectious disease caused by Mycobacterium tuberculosis, which infects the lungs (pulmonary TB) and other organs (extrapulmonary) such as the spine, brain, abdomen, lymph nodes, skin and joints. Drug resistant-TB, especially multidrugresistant TB (MDR-TB) drug-resistant-TB, has become a major public health worry across the globe. According to the 2014 WHO Global Report, of the total estimated 9 million TB cases in the world, approximately $56 \%$ were from South-East Asia and Western Pacific regions. Of these cases, 3\% of new cases and $21 \%$ of retreatment cases were attributed to MDR TB.

Multi-drug-resistant tuberculosis (MDR-TB) is a significant emerging public health worry of great importance worldwide. The current era of tuberculosis has been characterized by a startling increase in the number of MDR-TB cases, which causes higher mortality rates than drug-sensitive tuberculosis. ${ }^{1}$

Tuberculosis infection is still a major public health worry in India. India accounts for about $25 \%$ of the global TB burden. Worldwide, India is the country with the highest burden of both TB and MDR-TB. It has become very important to detect the antibiotic resistance among Mycobacterium tuberculosis strains with the worldwide recognition of strains which are resistant to anti-TB drugs and their adverse impact on clinical consequences. Globally, the burden of MDR-
TB has not been well assesed; however, India has the highest number of tuberculosis cases and most of these cases are MDR-TB. ${ }^{2}$

Several reports showed high drug resistance among pulmonary TB cases in India (Mathur et al., 2000; Hemvani et al., 2001; Paramsivan et al., 2002). One of the most critical challenges to health worldwide is the control of tuberculosis (TB). ${ }^{3}$ The recent emergence of strains of Mycobacterium Tuberculosis which are resistant to the major anti-tuberculosis drugs pose a threat to efforts to control this menace. TB predominantly affects the poor with over $80 \%$ of cases occurring in Asia and Africa. ${ }^{4}$ Although the greatest numbers of patients live in countries with a very high population in Asia, the highest incidence of disease is found in the WHO region of Africa. ${ }^{5}$

Drug-resistant tuberculosis particularly MDR-TB, was higher in patients with incomplete anti-tuberculosis treatment previously. ${ }^{6}$ A high level of drug resistance among the TB patients poses a threat of transmission of resistant strains to susceptible persons in the community. ${ }^{7}$ For this reason, attention towards the completion of anti-TB treatment are needed by proper patient counseling.

Drug-resistant tuberculosis (DR-TB) was reported during the period of introduction of anti-TB chemotherapy, but recently, extensively drug-resistant.

TB (XDR-TB) has created a threat to the global control of TB. The concept of XDR-TB was observed 
at the Centers for Disease Control and Prevention (CDC) in 2005. Subsequently, data on the resistance to second line drugs was seen during an outbreak at KwaZulu-Natal, South Africa, leading to increased mortality among TB cases which were co-infected with HIV in 2006.

The worldwide emergence of XDR-TB has raised concerns that the existing drug susceptible TB will be replaced by drug resistant TB with very limited treatment strategies. The rise of XDR-TB can be prevented by proper management with second line drugs (SLDs) in rifampicin-resistant TB (RR-TB) and multidrug-resistant TB (MDR-TB) cases.

Emergence of extensively drug-resistant tuberculosis (XDR-TB) has threatened to derail the efforts to control TB, especially in regions endemic to HIV. Managing XDR-TB is a challenge and understanding the risk factors and epidemiology associated with it is of prime importance in reducing this menace. The WHO report on XDR-TB from India has reported a variation from 0.3 to 60 per cent of MDR-TB.

XDR-TB emergence can be prevented to a great extent by early diagnosis and management in rifampicin-resistant TB (RR-TB) as well as multidrugresistant TB (MDR-TB) patients with second line antiTB drugs. Proper treatment of RR-TB, MDR-TB and XDR-TB cases only will not be enough to reduce the global burden in future. The Revised National Tuberculosis Control Programme and other global anti tuberculosis programmes need to prioritize on policies which focus on the effective as well as the judicious use of first-line anti-TB drugs in all diagnosed drug susceptible TB patients to prevent development of drug resistance.

\section{Materials and Method}

A record based Cross Sectional Study Design was carried out on patients visiting the Dept of Respiratory Medicine at a Tertiary Care Hospital in Western Maharashtra._Epidemiological data of TB patients and data on M. Tuberculosis resistance to first and second line TB drugs were retrospectively collected from 01 January 2012 to 31 December 2017.

Drug sensitivity to first and second line Anti- tubercular drugs was done by MIGIT method. Data was collected from Mycobacterium Tuberculosis Master Record Register at the Dept of Pathology at a Tertiary Care Hospital in Western Maharashtra. Patients giving inadequate samples and Patients giving defective sample in lab were excluded from the study. Analysis of various variables in the study was done after collection of relevant data, using appropriate statistical inferential procedures. Data was then entered and analyzed in Statistical Package for the Social Sciences (SPSS) version 22. Descriptive statistics was used to describe the distribution of all variables.

\section{Results}

Drug sensitivity to first and second line Antitubercular drugs was done by MIGIT method. Records of 734 sputum samples were collected from 701 (95.5\%) males and $33(4.5 \%)$ females were studied. Percentages of resistance to individual first and second line anti -TB drugs are shown in Fig.1. The drug sensitivity profile of these 13 drugs were evaluated and recorded.

Out of 734 sputum samples, 160 showed monoresistance i.e. resistance to one first-line anti TB drugs (INH, RIF, SM, PYZ and EMB) and the remaining 574 were sensitive to all the five first-line anti TB drugs.

Out of 734 isolates, 56 (7.62\%) showed multi drug resistance (MDR) i.e. resistance to at least INH and RIF whereas $39(5.6 \%)$ out of the 734 samples showed extensive drug resistance (XDR) i.e. Resistance to both INH and RIF, in addition to resistance to fluoroquinolones and to at least one injectable second line drugs. The trends in drug resistance showed a significant spike in the resistance to all First line and Second Line Anti Tuberculosis drugs in 2016.

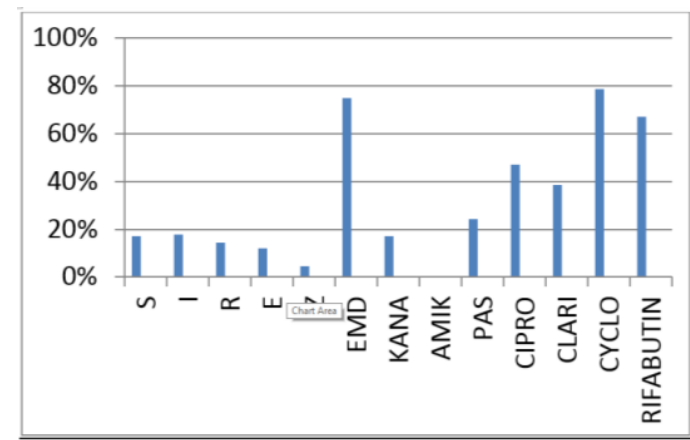

Fig. 1: Resistance to individual drugs used in this study

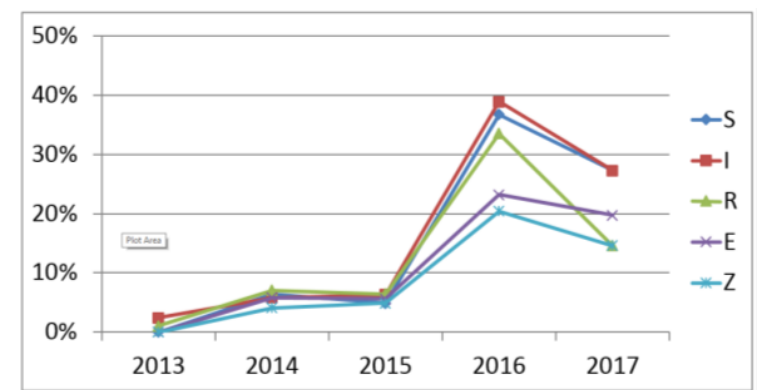

Fig. 2: Trends in First Line Drug Resistance from 2013-2017 


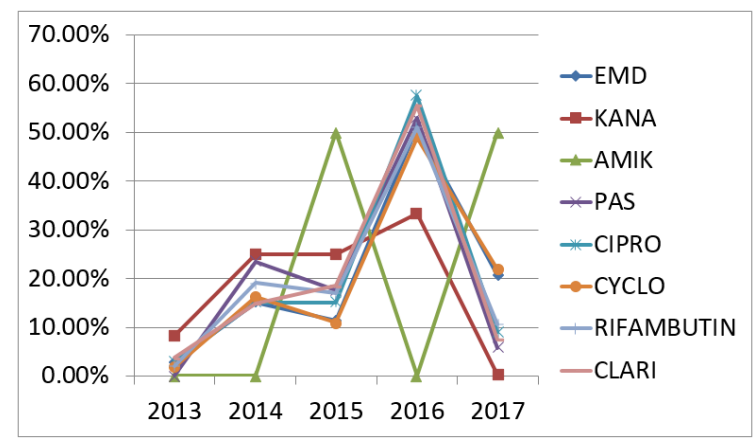

Fig. 3: Trends in Second Line Drug Resistance from 2013-2017

\section{Discussion}

This retrospective study done on drug-resistance patterns among tuberculosis cases has created valuable information in the context of the situation of drugresistant TB in India. Development of drug resistance $\mathrm{TB}$, including MDR, is attributed to poor compliance of patient ,inappropriate TB drug regimen, inadequate laboratory facilities for drug-susceptibility testing, and

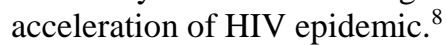

Resistance to INH and INH plus Rifampicin was $6.2 \%^{10}$ and $13.1 \%^{10}$ in India respectively whereas Shah et al (2002) studied 482 previously treated pulmonary tuberculosis patients and reported that resistance to INH and INH plus Rifampicin was $12.86 \%$ and $15.77 \%$, respectively. In our study, resistance to INH and INH plus Rifampicin was $17.6 \%$ and $7.6 \%$, respectively. Although resistance to INH was higher than in the study by Shah et al. (2002), INH plus RIF resistance was lesser.

Vijay et al reported that resistance to INH, either alone or in combination with another drug, was highest, at $27.4 \%$. SM resistance in India was $6.8 \%$ which was much lower than the $16 \%$ found in their study and corroborated with the present report. Resistance against Rifampicin in India was $5.1 \%{ }^{10}$ whereas it was $14.28 \%$ in the present study but was lower than the resistance to Rifampicin (15.5\%) reported in a previous study (Shah et al, 2002).

Non-compliance in patients is an important factor in this regard. Secondly, we found the highest number of resistance $(78.51 \%)$ against cycloserine among second-line drugs. The reason for this resistance may be attributed to the cross-resistance among drugs, e.g. cross-resistance of INH with ethionamide. This study shows a low resistance to fluoroquinolones among the strains which were tested in this study. In Indian scenario, this study underlines the necessity of direct observed therapy. .

Globally India has one of the highest cases of MDR-TB, with an estimated 63,000 patients having MDR strains among pulmonary TB cases in 2013, which accounts for more than 20 percent of the estimated global burden of drug resistant tuberculosis. ${ }^{9}$
In India, the incidence of multi drug resistant tuberculosis (MDR) is 3.4\%. A high degree $(7.62 \%)$ of MDR-TB was found among patients in this study. However, $462(63 \%)$ isolates were sensitive to all four first-line drugs (INH, RIF, SM and EMB) that were tested. Although non-compliance is a major factor for poor outcome of therapy, this study underscores the presence of MDR-TB strains among these patients.

All MDR-TB patients should be tested for XDRTB in high-resource settings. The case detection also depends on the availability of SLDs. According to the WHO, in areas with limited second-line DST, patients should be tested at the very least if suspected to have risk factors for XDR-TB.

Further, in areas where there is no capacity for second-line DST exists, this investigation has to be done from a reliable SRL. Individuals presumed to have XDR-TB should have DST to H, R, three SLIDs (Km, Amk and $\mathrm{Cm}$ ) and FQs. The routine testing of DST for SLDs is not possible due to its cost, difficulty and poor reliability Bedaquiline is the approved drug for the treatment of MDR-TB and XDR-TB by the WHO, which is of the diarylquinoline class of antibiotics. ${ }^{12}$ Bedaquiline has a very high bactericidal activity against drug resistance which has been proved on the basis of a multicentric phase-II trial.

Various measures have to be undertaken while treating MDR-TB and XDR-TB patients for better results such as respiratory infection control, prompt hospitalization in poor general condition of the patient or coexistence of comorbidities, provision of proper shelter if the patient's social condition prevents proper home care, management of HIV co-infection, proper monitoring and complete social support for proper adherence, full access to palliative services and focus on patient-centred approach.

\section{Conclusion}

Our study confirms that drug resistance, which includes multi drug resistance( MDR) and extensively drug resistance (XDR) observed against all first-line TB drugs was a real threat in the management of TB infection. The patterns of resistance which were observed in this study could definitely assist the clinicians in providing appropriate treatment regimen to TB patients and improve their clinical outcome.

The trends in TB drug resistance patterns emphasize the need for individualized drug regimens, structured on the basis of full drug susceptibility results to first and second line anti-TB drugs. A drug-resistant TB case-finding strategy, based only on techniques such as GeneXpert that identify only TB with resistance to Rifampicin , may lead to suboptimal treatment regimens for patients suffering from disease due to preXDR-TB strains, and may lead to amplification of the resistance and spread of further worse forms of drugresistant TB within the community. 
One of the top most priorities is to diagnose MDRTB and XDR-TB in clinical practice by increasing the clinical laboratory capacity throughout the country. All reference laboratories in the country should be able to perform high quality conventional DST for all the second line drugs(SLDs) to diagnose XDR-TB effectively. Molecular tests to identify $H$ and $R$ resistance together along with DST for SLDs will help in rapid diagnosis, but there is a need of large inputs in training personnel and and provide facilities to make it feasible in a resource-limited country like India. ${ }^{11} \mathrm{New}$ research and development in the areas which involve application of molecular biology in epidemiology could help in understanding of the mechanisms which lead to drug resistance.

The proper management of XDR-TB depends on judicious use of SLDs to reduce the morbidity and the mortality and transmission based on the present situation. Optimal treatment of RR-TB, MDR-TB and XDR-TB cases alone will not reduce the global burden. The TB control programmes will have to emphasize on the policies which focus on the effective use of firstline drugs in every new patient so as to prevent the emergence of MDR-TB, XDR-TB and XXDR-TB or TDR-TB.

\section{References}

1. The World Health Organization. Global tuberculosis control: surveillance, planning, financing.WHO report 2008. Geneva

2. Udwadia ZF, "Totally drug resistant-tuberculosis in India: The bad just got worse". JACP, 2016; 4(2):41-42.

3. Sandhu, Gursimrat K. "Tuberculosis: Current Situation, Challenges and Overview of Its Control Programs in India." Journal of Global Infectious Diseases 3.2 (2011): 143-150. PMC. Web. 10 Feb. 2018.

4. Zager, Ellen M, and Ruth McNerney. "MultidrugResistant Tuberculosis." BMC Infectious Diseases 8 (2008): 10. PMC. Web. 10 Feb. 2018.

5. Corbett et al,'Tuberculosis in sub-Saharan Africa: opportunities, challenges, and change in the era of antiretroviral treatment'. Lancet. 2006; 367:926-937.

6. Suhail A,"Recent advances in the diagnosis and treatment of multidrug-resistant tuberculosis". https://doi.org/10.1016/j.rmed.2009.07.010

7. Banu et al,PLoS One. 2012;7(7). doi: 10.1371/journal.pone.0040545. Epub 2012 Jul 11.

8. Cucanawangish et al," Mycobacterium tuberculosis resistance pattern against first-line drugs in patients from urban area". IJP,2016;4(4):302-305.

9. Dalal et al, "Resistance Patterns among Multidrug Resistant Tuberculosis Patients in Greater Metropolitan Mumbai: Trends over Time". PLOS one, DOI:10.1371/journal.pone.0116798 Jan 21, 2015

10. Goyal et al,BMC Public Health (2017) 17:817 DOI 10.1186/s12889-017-4779-5

11. Prasad et al, "Extensively drug-resistant tuberculosis in India: Current evidence on diagnosis \& management". Indian J Med Res 145, March 2017, pp 271-293.

12. Sloan DJ, Lewis JM. :Management of multidrug-resistant TB: Novel treatments and their expansion to low resource settings".Trans R Soc Trop Med Hygm2016;110:163-72.
13. World Health Organization.Companion handbook to the WHO guidelines for the programmatic management of drug-resistant tuberculosis. WHO/HTM/TB/2014.11. Geneva: WHO;2014. 